\title{
Linking DNA replication checkpoint to MBF cell-cycle transcription reveals a distinct class of G1/S genes
}

\author{
Francisco M Bastos de Oliveira ${ }^{1,4}$, \\ Michael R Harris ${ }^{2,4}$, Pijus Brazauskas², \\ Robertus AM de Bruin 2,3,* \\ and Marcus B Smolka ${ }^{1, *}$
}

${ }^{1}$ Department of Molecular Biology and Genetics, Weill Institute for Cell and Molecular Biology, Cornell University, Ithaca, NY, USA, ${ }^{2} \mathrm{MRC}$ Laboratory for Molecular Cell Biology, University College London, London, UK and ${ }^{3}$ The UCL Cancer Institute, University College London, London, UK

Reprogramming gene expression is crucial for DNA replication stress response. We used quantitative proteomics to establish how the transcriptional response results in changes in protein levels. We found that expression of G1/S cell-cycle targets are strongly up-regulated upon replication stress, and show that MBF, but not SBF genes, are up-regulated via Rad53-dependent inactivation of the MBF co-repressor Nrm1. A subset of G1/S genes was found to undergo an SBF-to-MBF switch at the G1/S transition, enabling replication stress-induced transcription of genes targeted by SBF during G1. This subset of G1/S genes is characterized by an overlapping Swi4/ Mbp1-binding site and is enriched for genes that cause a cell cycle and/or growth defect when overexpressed. Analysis of the prototypical switch gene TOS 4 (Target $\underline{O}$ $S B F \underline{4}$ ) reveals its role as a checkpoint effector supporting the importance of this distinct class of G1/S genes for the DNA replication checkpoint response. Our results reveal that replication stress induces expression of G1/S genes via the Rad53-MBF pathway and that an SBF-to-MBF switch characterizes a new class of genes that can be induced by replication stress.

The EMBO Journal (2012) 31, 1798-1810. doi:10.1038/

emboj.2012.27; Published online 14 February 2012

Subject Categories: chromatin \& transcription; cell cycle; genome stability \& dynamics

Keywords: G1-S transcription; Rad53; replication checkpoint; replication stress; SBF and MBF

\footnotetext{
*Corresponding authors. RAM de Bruin, MRC Laboratory for Molecular Cell Biology, University College London, Gower St, London, WC1E 6BT, UK. Tel.: + 440207679 7255; Fax.: + 440207679 7805;

E-mail: r.debruin@ucl.ac.uk or MB Smolka, Department of Molecular Biology and Genetics, Weill Institute for Cell and Molecular Biology, Cornell University, 339 Weill Hall, Ithaca, NY 14853-7202, USA.

Tel.: + 1607255 0274; Fax.: + 1607255 5961;

E-mail: mbs266@cornell.edu

${ }^{4}$ These two authors contributed equally to this work
}

Received: 14 December 2011; accepted: 20 January 2012; published online: 14 February 2012

\section{Introduction}

A cell division cycle is initiated during the G1 phase of the cell cycle by the transcriptional induction of genes that drive transition into $\mathrm{S}$ phase. In human cells, G1/S transcriptional regulation depends on the E2F family of transcription factors and their co-regulators, the transcriptional co-repressor pRB family members (Frolov and Dyson, 2004; Cobrinik, 2005; Chen et al, 2009). The importance of G1/S transcriptional regulation for controlled cell-cycle progression is illustrated by the fact that E2F-dependent cell-cycle transcription is misregulated in nearly all tumour types (Stevens and La Thangue, 2003).

In budding yeast, G1/S transcription depends on two transcription factor complexes, SBF and MBF (Breeden, 2003; Wittenberg and Reed, 2005). The SBF and MBF complexes comprise a shared regulatory subunit, Swi6, and the DNA-binding subunits Swi4 and Mbp1, respectively. Swi4 and Mbp1 recognize distinct DNA sequence elements, SCB

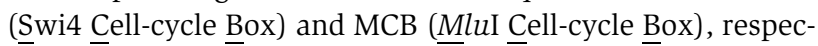
tively, which define SBF and MBF target promoters. SBF is a potent transcriptional activator, while MBF behaves primarily as a transcriptional repressor (Amon et al, 1993; Bean et al, 2005; de Bruin et al, 2006). During the cell cycle, SBF and MBF are bound and regulated by the transcriptional co-repressors, Whi5 and Nrm1, respectively (Costanzo et al, 2004; de Bruin et al, 2004, 2006).

Once G1/S transcription is activated, cells progress into $S$ phase and initiate DNA replication. During DNA replication, cells are particularly vulnerable to accumulate genomic instability, as replication forks are prone to stall and collapse when encountering replication blocks or damaged DNA templates (Lopes et al, 2001; Tercero and Diffley, 2001). To properly replicate the genome and avoid DNA damage, cells rely on the DNA replication checkpoint. In budding yeast, the DNA replication checkpoint is coordinated mostly by the Pl3like sensor kinase Mec1 and by the effector kinase Rad53, orthologues of human ATR and CHK2/CHK1, respectively (Segurado and Tercero, 2009). The replication checkpoint protects cells from irreversible DNA damage by delaying mitotic entry and, among other responses, initiating a transcriptional programme to deal with stresses in the replication process (Zhou and Elledge, 1993; Allen et al, 1994; Gasch et al, 2001; Zhao et al, 2001).

Genome-wide expression analysis in budding yeast has revealed that hundreds of genes are regulated in response to genotoxic stress (Fu et al, 2008). The best understood mechanism by which the DNA replication checkpoint regulates replication stress-induced transcription is via inactivation of the transcriptional repressor Crt1 (Huang et al, 1998). Upon replication stress, Rad53 phosphorylates and activates the downstream checkpoint kinase Dun1, which in turn phosphorylates the transcriptional repressor Crt1. Phosphorylated Crt1 
becomes inactive and no longer binds its target promoters leading to the transcription activation of DNA damage response (DDR) genes. However, the Rad53-Dun1-Crt1 regulatory circuit represents only a small part of the checkpoint transcriptional response and is not conserved in higher eukaryotes.

In order to establish how the transcriptional response results in the accumulation of proteins during replication stress, we utilized a quantitative proteomic approach. From this study we found that upon genotoxic stress, apart from Crt1 targets, expression of several G1/S cell-cycle genes are strongly up-regulated. We show that MBF transcripts are up-regulated in response to replication stress via Rad53dependent, but Dun1-independent, inhibition of the MBF co-repressor Nrm1. Detailed analysis of the putative SBFonly target TOS4 (Target $\underline{O} \underline{S B F} \underline{4}$ ) revealed an SBF-to-MBF switch at its promoter that allows its Rad53-dependent transcriptional induction upon replication stress. This led to the identification of a specific subset of G1/S genes that are likely targeted by SBF during G1 and undergo an SBF to MBF switch at the G1/S transition. These genes are characterized by an overlapping SBF and MBF-binding motif (SCB and MCB, respectively), which likely results in a competition for SBF/ MBF binding and might be the basis of the SBF-to-MBF switch. This new set of G1/S genes is regulated differently in many ways as compared with G1/S target genes containing non-overlapping SCB and MCB motifs in their promoter. These switch G1/S genes are enriched for genes that cause a cell cycle and/or growth defect when overexpressed and include genes important for the checkpoint response. These findings define the mechanism of the regulation of cell-cycle genes in response to DNA replication stress and reveal a novel mode of transcriptional regulation via the coordinated action of SBF and MBF at distinct stages of the cell cycle.

\section{Results}

\section{Expression of G1/S genes is induced during replication stress}

Treating cells with hydroxyurea (HU), a specific inhibitor of ribonucleotide reductase, effectively decreases dNTP levels, resulting in the stalling of DNA replication forks and activation of the DNA replication checkpoint response. In order to establish which proteins accumulate in response to DNA replication stress, we carried out quantitative proteomics analysis comparing the protein levels of cells before and after HU-induced replication stress (Dataset 1 in Supplementary data). As expected, we detected an increase in the abundance of the well-established replication stress-induced proteins Rnr3 and Hug1, which are under the control of the Crt1 transcription repressor (Figure 1A). In addition, we found increased abundance of the MBF-only target Rnr1 as well as of other G1/S proteins, including Mcd1 (putative MBF-only target), Tos4 (putative SBF-only target) and Ndd1 (SBF-only target), in response to replication stress. Increase on Tos4 protein levels during replication stress was also validated by western blot analysis (Supplementary Figure S1). To establish whether the accumulation of these proteins during DNA replication stress is the result of transcriptional induction, we carried out a gene expression analysis during the cell cycle in untreated and HU-treated cells (Figure 1B). Treatment with other genotoxic agents (MMS and CPT) has been shown to give similar results
(Wittenberg laboratory, personal communication). This analysis confirms previous observations showing transcriptional induction of RNR3 and HUG1 strictly in response to DNA replication stress. It also shows that expression of the G1/S genes TOS4, RNR1 and MCD1 is periodic during an unperturbed cell cycle, peaking at the G1/S transition and upregulated during $S$ phase in response to replication stress. Transcription of NDD1 was not increased during replication stress (Supplementary Figure S2), revealing that the abundance of Ndd1 protein in response to replication stress is under an alternative mode of regulation.

Together, these data show that both the transcript and protein levels of the G1/S cell cycle regulated genes TOS4, MCD1 and RNR1 are induced in response to DNA replication stress. The fold induction observed for these proteins in our proteomic analysis is among the most drastic changes observed in the proteome, supporting that G1/S transcription is strongly impacted upon replication stress. Moreover, the transcriptional regulation of G1/S genes is different from that of Crt1 targets, whose transcription is not induced in a normal cell cycle but strongly up-regulated in response to DNA replication stress.

\section{Replication stress induces expression of G1/S genes via a Rad53-dependent but Dun1-independent pathway}

In response to DNA replication stress, Dun1-dependent phosphorylation inactivates the transcriptional repressor Crt1 leading to induction of its targets, such as RNR3 and HUG1. To establish whether the induction of the G1/S genes TOS4, MCD1 and RNR1 during replication stress depends on Dun1, we measured their transcript levels during the cell cycle and in response to $\mathrm{HU}$ treatment in wt and dun $1 \Delta$ cells. As previously shown, the induction of the Crt1 targets RNR3 and HUG1 in response to replication stress depends on Dun1 (Figure 1C, upper panels). We also show that G1/S targets remain cell cycle regulated and replication stress induced in dun $1 \Delta$ cells, revealing that their expression is Dun1 independent (Figure 1C, upper panels). Upstream of Dun1 functions the Rad53 protein kinase. To determine if the expression of TOS4, MCD1 and RNR1 is dependent on Rad53, the experiment above was carried out in rad53 $\Delta$ cells. Since Rad53 is an essential gene, the experiment was done in an sml1 $\Delta$ background, which suppresses the lethality of Rad53 inactivation. The expression analysis establishes that, as expected, the induction of Crt1 targets is Rad53 dependent (Figure 1C, lower panels). It also shows that the induction of TOS4, MCD1 and RNR1 depends on Rad53. Overall, these data show that the induction of the G1/S cell cycle regulated genes TOS4, MCD1 and RNR1, in response to replication stress is Rad53 dependent, but is independent of Dun1.

\section{Targets of MBF, but not SBF, are up-regulated in response to replication stress}

To determine if transcriptional induction of G1/S genes upon replication stress is a general feature of G1/S genes or is specific for MBF and/or SBF-only targets, we determined the cell-cycle expression levels of other well-established G1/S transcripts, two SBF targets (SVS1 and CLN2) and one MBF target $(C D C 21)$, in untreated and HU-treated conditions. Our analysis revealed that whereas the SBF targets SVS1 and CLN2 are repressed in a timely manner, the MBF target $C D C 21$ is induced in response to DNA replication stress 

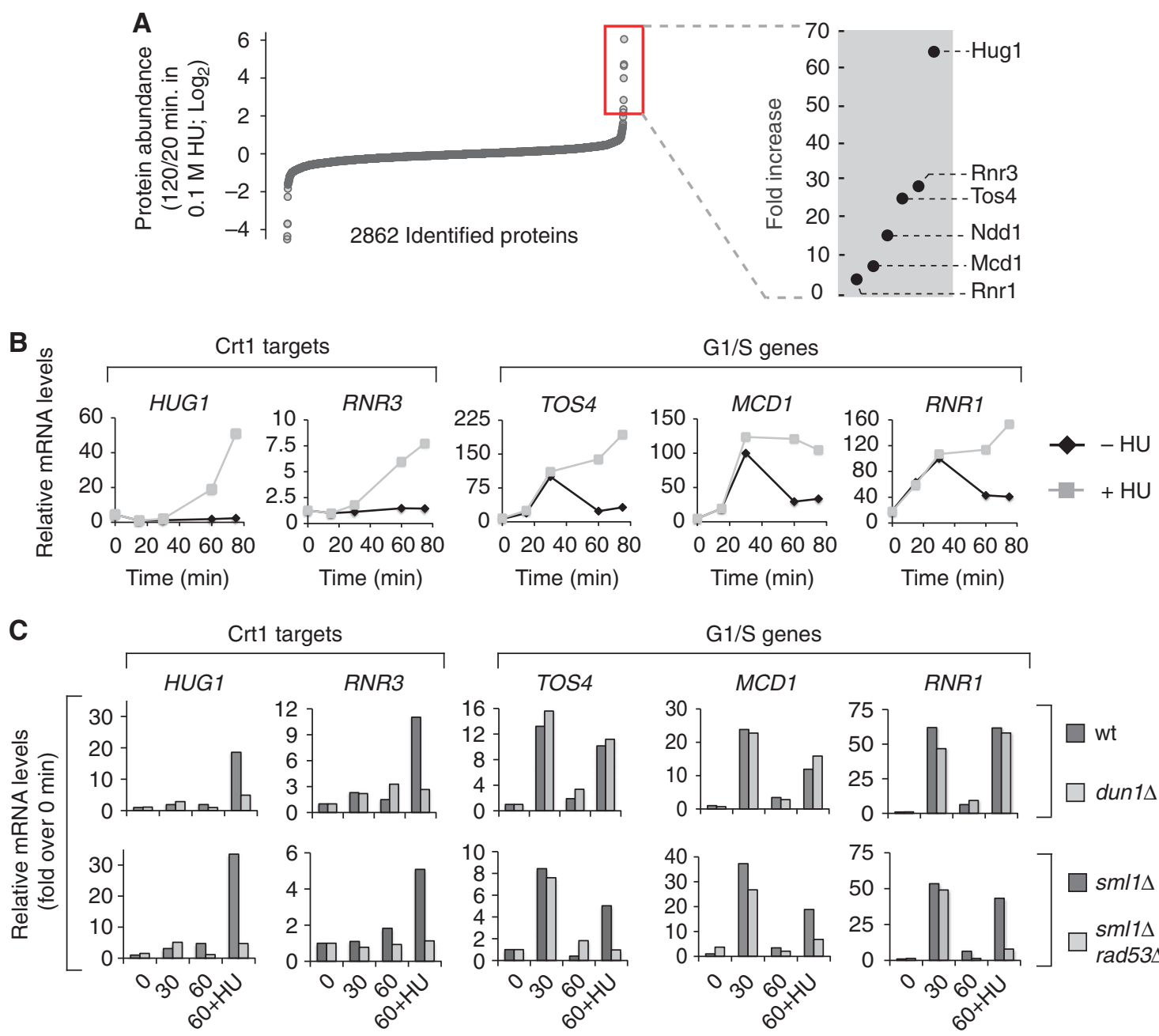

rt1 targets

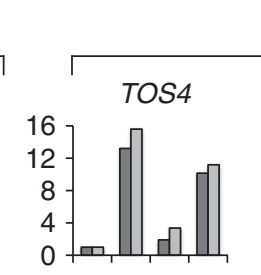

G1/S genes
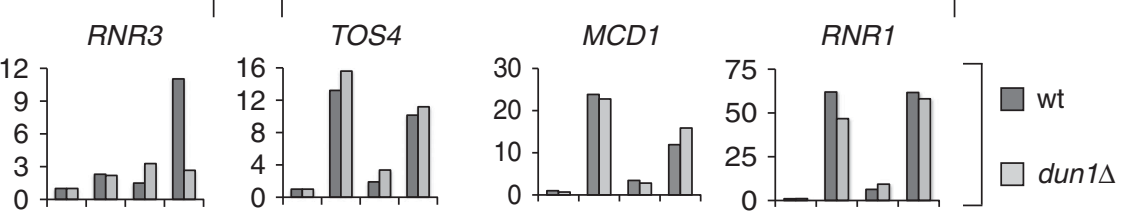

Time (min)
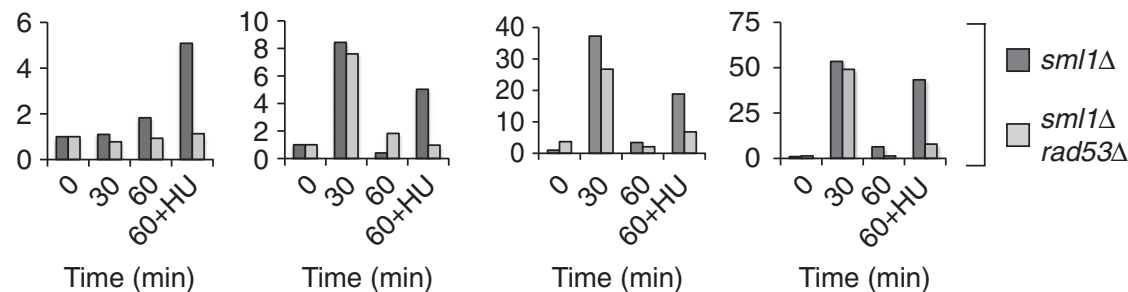

Figure 1 Replication stress-induced reprogramming of gene expression includes transcriptional up-regulation of G1/S genes. (A) Proteomic analysis of changes in protein abundance following replication stress. Cells were arrested in G1 and released for 20 or 120 min in media containing $100 \mathrm{mM}$ HU. Rad53 is activated at $\sim 25 \mathrm{~min}$ after release from G1 arrest. Shown results are the average ratio (120/20 min) for each of the 2862 proteins identified and quantified in the experiment. (B) Relative mRNA levels of indicated genes in cells synchronized by $\alpha$-factor arrest and release, in the presence or absence of HU. Transcript levels are represented as a fold over 0 min for the Crt1 targets and a fold over max. unt. $100 \%$ for the G1/S targets. (C) Relative mRNA levels of indicated genes in wild-type, dun $1 \Delta$ or sml1 $/ \mathrm{rad} 53 \Delta$ cells. Expression levels, at indicated time points ( $\mathrm{min}$ ), are presented as fold induction of levels detected in time 0 . For samples treated with $\mathrm{HU}, 100 \mathrm{mM} \mathrm{HU}$ was added 20 min after release from $\alpha$-factor arrest. Levels of mRNA were quantified by RT-qPCR and normalized to ACT1.

similarly to what we observed for RNR1, MCD1 and TOS4 (Figure 2A). Furthermore, expression of $C D C 21$ during replication stress was found to depend on Rad53 but not on Dun1 (Figure 2B). These data reveal that MBF-dependent, but not SBF-dependent, cell-cycle transcripts are induced in response to DNA replication stress in a Rad53-dependent manner. Interestingly, TOS4 was initially identified as a target of SBF (Iyer et al, 2001); however, its pattern of expression during replication stress suggests that TOS4 is likely regulated by MBF during $S$ phase.

\section{SBF is replaced by MBF at the promoter of TOS4 during the G1/S transition}

To determine if TOS4 is regulated by MBF during any stage of the cell cycle, we performed ChIP analysis, pulling down myc-tagged Swi4 and Mbp1 proteins and examining their binding to the TOS4 promoter at different time points after release from G1 arrest. As shown in Figure 3A, we found that
Swi4 binds the TOS4 promoter in G1, but once cells progress into S phase, Swi4 leaves the TOS4 promoter and Mbp1 binds it. These findings suggest that SBF and MBF regulate TOS4 expression in a mutually exclusive manner at different stages of the cell cycle, a feature that differentiates TOS4 from G1/S genes regulated by SBF-only (i.e., SVS1), MBF-only (i.e., CDC21) or dual-regulated SBF and MBF (i.e., CLN1). This transcription factor switching at G1/S promoters during the cell cycle has not been observed in yeast before but resembles the mode of regulation in human cells for the E2F transcription factors at G1/S promoters (Dimova and Dyson, 2005; Moon and Dyson, 2008).

\section{TOS4 transcription is activated by SBF during G1 and repressed by MBF during $S$ phase}

Previous work has shown that whereas SBF functions primarily as a transcriptional activator during the G1 phase of the cell cycle, MBF functions as a transcriptional repressor 

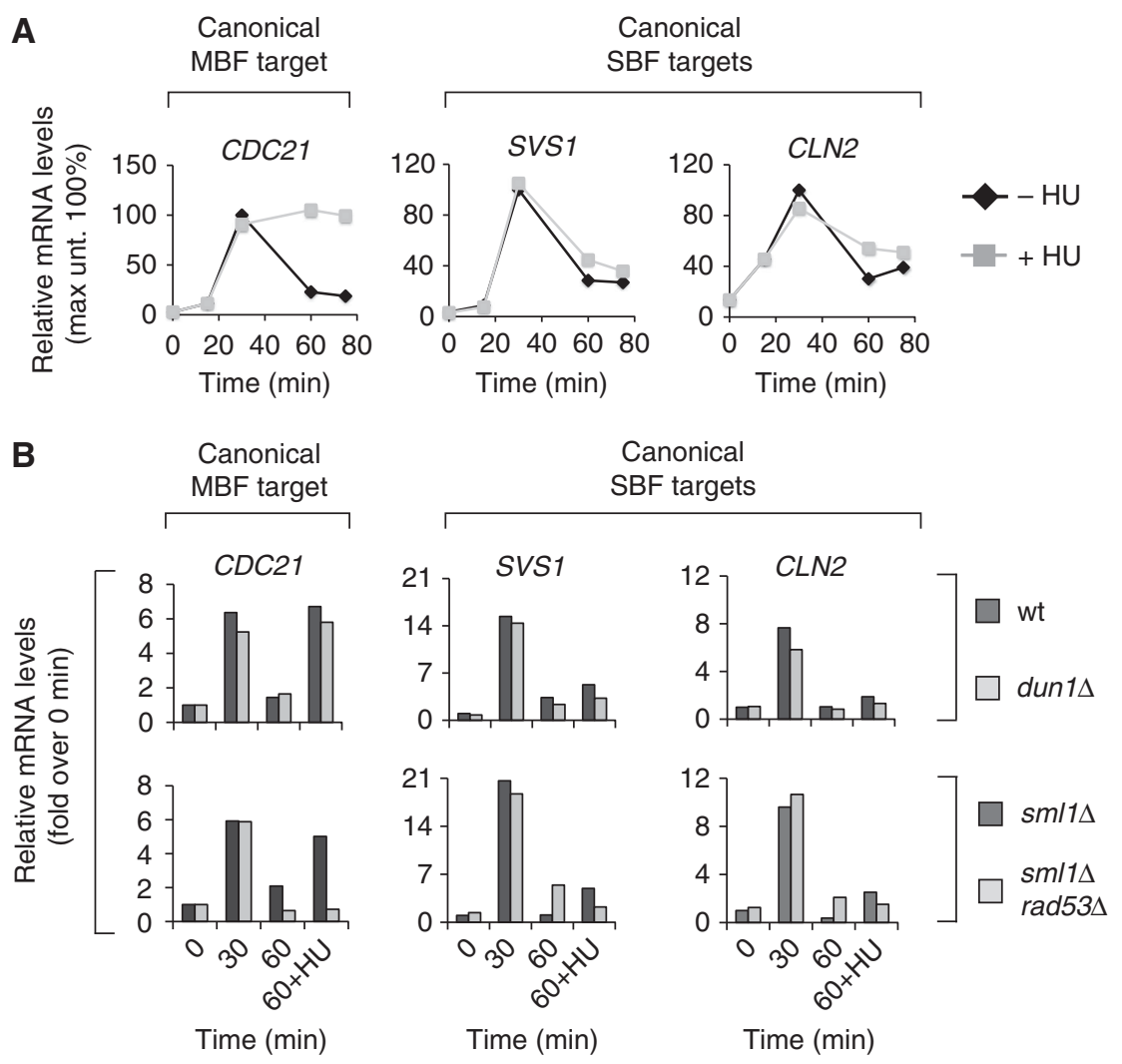

Figure 2 Expression of canonical MBF and SBF targets during replication stress. (A) Relative mRNA levels of the canonical SBF targets SVS1 and $C L N 2$, and of the canonical MBF target CDC21 in cells synchronized by $\alpha$-factor arrest and release, in the presence or absence of HU Transcript levels are represented as a percentage of highest level (100\%) observed in untreated samples. (B) Relative SVS1, CLN2 and CDC21 mRNA levels in wild-type, dun $1 \Delta$ or $\operatorname{sml} 1 \Delta / \operatorname{rad} 53 \Delta$ cells. The experiment was performed as described in Figure 1C.

outside of G1 (Amon et al, 1993; Bean et al, 2005; de Bruin et al, 2006). To determine the role of MBF and SBF in controlling the expression of TOS4 during the cell cycle, we analysed the pattern of TOS4 transcription in wild-type cells and in cells lacking either Mbp1 (MBF $\Delta$ ) or Swi4 (SBF $\Delta$ ). The transcription profile of the well-established SBF-only target, SVS1, MBF-only target, CDC21 and, the dual-regulated SBF and MBF target, CLN1 were used as controls (Bean et al, 2005; de Bruin et al, 2006; Eser et al, 2011). As expected, deletion of either MBP1 or SWI4 had no effect on the periodic expression of SVS1 and CDC21, respectively. In turn, as previously shown, deletion of SWI4 abrogated the transcriptional induction of SVS1 and its periodicity and deletion of MBP1 abolished periodicity and de-represses expression of CDC21 throughout the cell cycle (Figure 3B). Surprisingly, TOS4 transcription pattern showed characteristics of both SVS1 and CDC21 genes (Figure 3B). Our data show that deletion of SWI4 significantly decreases TOS4 peak expression levels during G1/S, confirming that TOS4 is indeed under the control of SBF (Figure 3B). In contrast, while TOS4 transcript levels in mbp1 $1 \Delta$ cells were comparable to wild-type levels during G1, it was significantly de-repressed outside of G1. Finally, we show that CLN1, a dual-regulated SBF and MBF target, behaves like an SBF target, loosing its transcriptional periodicity in swi4s cells. Together, these results reveal that while SBF is important for the transcriptional activation of TOS4 during G1, MBF represses TOS4 expression outside of G1.

\section{During G1, Swi4 competes with Mbp1 for binding to the TOS4 promoter}

Our findings show that TOS4 is regulated by SBF during G1 and by MBF outside of G1. SBF and MBF targets are traditionally defined by the presence of SCB (CRCGAAA) and MCB (ACGCGN) DNA regulatory elements in the promoter sequences, respectively. In line with our observations, the TOS4 promoter contains both an MCB and SCB element (Figure 4A). Interestingly, the sequence of the MCB and SCB elements partially overlaps, which could represent the basis of a competition mechanism between SBF and MBF binding for the TOS4 promoter. This feature is markedly different from the classical dual-regulated SBF and MBF target CLN1 that contains SCB and MCB regulatory elements that do not overlap. To examine if Swi4 and Mbp1 compete for binding to the TOS4 promoter, we carried out ChIP experiments pulling down myc-tagged Swi4 and Mbp1 proteins in wt and mpb1 $\Delta$ and swi4 $\Delta$ non-synchronized cell cultures, respectively. Figure 4B shows that the TOS4 promoter is mainly bound by Swi4; however, whereas inactivation of Mbp1 has no effect on Swi4 binding, in the absence of Swi4, a significant increase in binding of Mbp1 to the TOS4 promoter can be observed. The increase in Mbp1 binding to the TOS4 promoter in the absence of Swi4 is mainly the result of enhanced binding during the G1 phase of the cell cycle, when SBF normally binds (Figure 4C). These data indicate that Swi4 competes with Mbp1 for binding to the TOS4 promoter during G1 of the cell cycle. These results suggest that the cell cycle- 
A

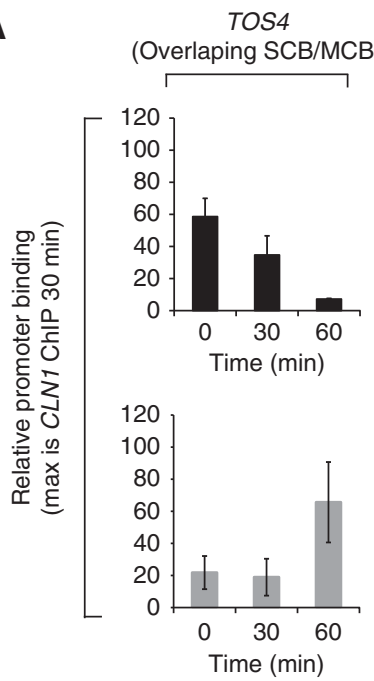

B

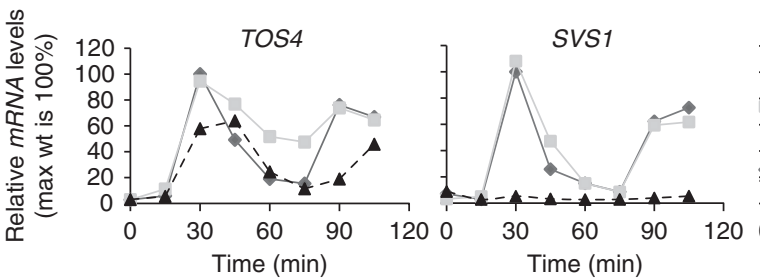

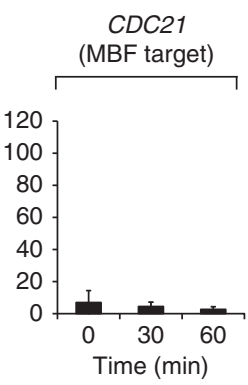
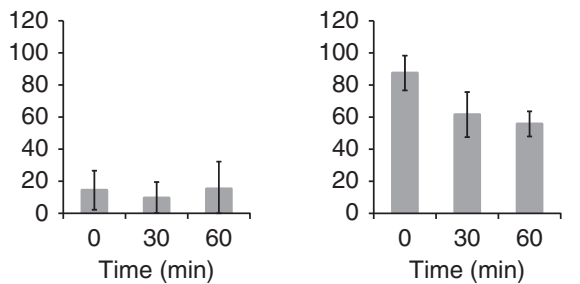

CLN1
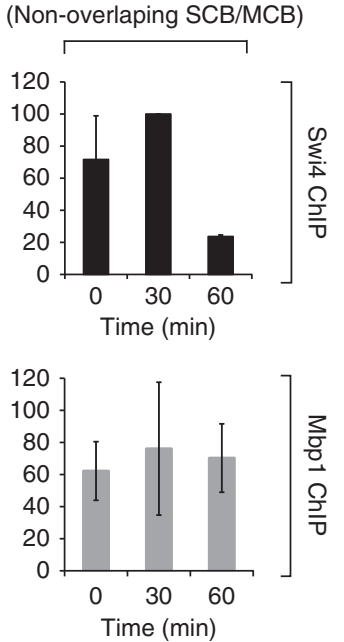

Figure 3 An SBF-to-MBF switch at the promoter of the G1/S gene TOS4. (A) ChIP analysis for Swi4-myc or Mbp1-myc at the TOS4, SVS1, $C D C 21$ and $C L N 1$ promoters during the G1 and S phase of the cell cycle. Analysis was performed in G1 synchronized cells released from $\alpha$-factor arrest and samples were taken at the indicated intervals. (A) Enrichment levels were accessed by qPCR and are presented as a percentage of highest obtained level in the separate Swi4 ChIPs and Mbp1 ChIPs after normalization to whole cell extract (WCE) levels. Average value and error bars are derived from biological replicates. (B) Relative mRNA levels of indicated genes during the cell cycle in wild-type, swi4s and $\operatorname{mbp} 1 \Delta$ cells. Cells were synchronized in G1 by $\alpha$-factor, released from the arrest and collected at the indicated times after release. Levels of mRNA were quantified by RT-qPCR and normalized to ACT1. Transcript levels are represented as a percentage of highest level (100\%) observed in wild-type cells.

dependent switch between SBF and MBF at the promoter of TOS4 is governed by a competition between Swi4 and Mbp1 for the overlapping MCB/SCB motifs present in the TOS4 promoter.

\section{During S phase, the replacement of SBF by MBF on the TOS4 promoter is likely an active process}

During G1/S transition, active $\mathrm{Clb} / \mathrm{CDK}$ inhibits the ability of Swi4 to bind its target promoters and shuts off SBF-dependent transcription. To determine if MBF replaces SBF at switch promoters during $\mathrm{S}$ phase simply because SBF leaves promoters, or if MBF actively replaces SBF we have carried out a cell-cycle experiment using cells that overexpress a hyperstable form of the Clb/CDK-specific inhibitor Sicl (sic1 $\Delta \mathrm{p})$ in order to keep $\mathrm{Clb} / \mathrm{CDK}$ inactive. It has been shown that under these conditions SBF-only genes remain active whereas MBFonly genes are still inactivated in Nrm1-dependent manner (de Bruin et al, 2006). Therefore, if MBF replaces SBF at switch promoters during $S$ phase simply because SBF leaves promoters in a $\mathrm{Clb} / \mathrm{CDK}$-dependent manner, switch genes should remain active in the absence of $\mathrm{Clb} / \mathrm{CDK}$ activity. However, if MBF actively removes SBF to replace it from switch gene promoters during $\mathrm{S}$ phase, switch genes should still be repressed in the absence of $\mathrm{Clb} / \mathrm{CDK}$ activity. As expected, our data show that overexpression of $\operatorname{Sic} 1 \Delta \mathrm{p}$ had no effect on the periodic expression of MBF-only targets (i.e., RNR1) but increases the expression of SBF-only targets (i.e., CLN2) and dual-regulated SBF and MBF targets (i.e., CLN1).
Interestingly, TOS4 expression was shown to be inactivated in a timely manner even in the absence of $\mathrm{Clb} / \mathrm{CDK}$ activity (Figure 4D). This result suggests that MBF can actively replace $\mathrm{SBF}$ at switch genes promoters during $\mathrm{S}$ phase.

\section{Rad53 mediates induction of TOS4 and other MBF targets via inactivation of the co-repressor Nrm1}

Our findings show that due to an SBF-to-MBF switch at the G1/ $\mathrm{S}$ transition, expression of TOS4 during $\mathrm{S}$ phase is repressed by MBF. Upon replication stress, Rad53 de-represses TOS4 expression. Previous studies have shown that transcriptional repression of MBF targets, as cells exit G1 phase, depends on a negative feedback loop involving the transcriptional co-repressor Nrm1 (de Bruin et al, 2006). Nrm1, an MBF target itself, accumulates during the G1/S transition and binds to MBF at promoters to repress MBF-dependent transcription. The role of $\mathrm{Nrm} 1$ in the regulation of MBF target genes positions it as a potential target through which the replication checkpoint could activate MBF transcription at the G1/S transition. We therefore investigated whether Nrm1 function is regulated in response to DNA replication stress induced by HU treatment.

We reasoned that if $\mathrm{Nrm} 1$ is inactivated as part of the checkpoint response then MBF transcripts levels in $\mathrm{nrm} 1 \Delta$ cells should resemble those of HU-treated cells. In addition, HU treatment of $n r m 1 \Delta$ cells should not further induce MBFdependent genes. Our expression analysis shows that both assumptions hold true. MBF targets, including TOS4, are de- 
A

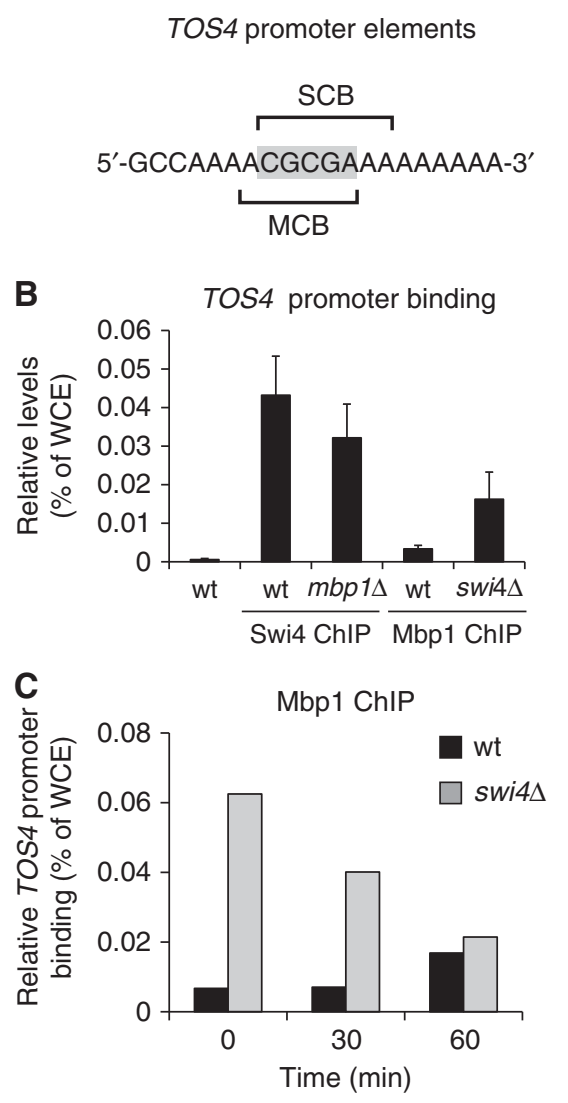

D $-w t$

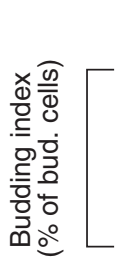

- GAL-Sic1 $\Delta \mathrm{p}$

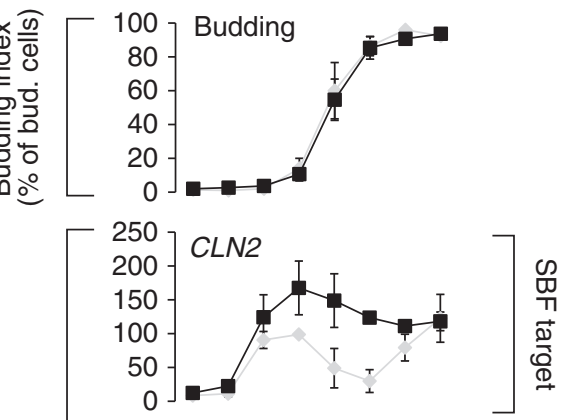

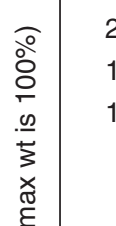
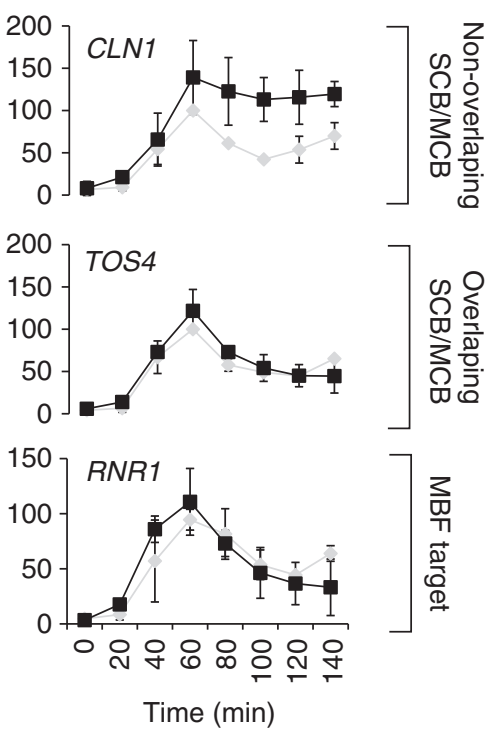

Figure $4 \mathrm{SCB} / \mathrm{MCB}$ overlapping motifs at G1/S promoters provides the rationale for SBF and MBF competition. (A) Schematic representation of overlapping SBF (SCB) and MBF (MCB) DNA-binding motifs identified in the TOS4 promoter element $(-232$ to -214 from the ATG start codon). Grey shade represents SCB/MCB overlapping region. (B) ChIP analysis for Mbp1-myc and Swi4-myc at the TOS4 promoter in wildtype, swi $4 \Delta$ and $m b p 1 \Delta$ cells. Analysis was performed in asynchronous cells and enrichment levels were accessed by qPCR and are shown as a percentage of WCE signal. Untagged cells were included as negative control. Average value and error bars are derived from experimental triplicates. (C) ChIP analysis for Mbp1-myc at TOS4 promoter in wild-type and swi4A cells. Experiment was performed as described in Figure 3A. (D) Relative mRNA levels of indicated genes during the cell cycle in wild-type, and cells expressing sic1 $\triangle \mathrm{p}$ from the inducible GAL1 promoter. Cells were synchronized in G1 by $\alpha$-factor, released from the arrest as detailed in Materials and methods and collected at the indicated times after release. Levels of mRNA were quantified by RT-qPCR and normalized to ACT1. Transcript levels are represented as a percentage of highest level (100\%) observed in wild-type cells. Budding index (\% budded cells) was measured as an indicator of synchrony. Average value and error bars are derived from biological triplicates.

repressed during S phase in $n r m 1 \Delta$ cells comparable to levels observed in HU-treated wild-type cells (Figure 5). In addition, $\mathrm{HU}$ treatment of $\mathrm{nrm} 1 \Delta$ cells does not significantly induce MBF transcripts. As expected, the Crt1 target HUG1 is still induced in response to HU treatment in $n r m 1 \Delta$ cells. Furthermore, whereas Nrm1 is able to bind and repress the MBF-dependent TOS4, RNR1 and CDC21 promoters during S phase, HU-induced DNA replication checkpoint activation results in loss of Nrm1 from these promoters (Supplementary Figure S3). Overall, these data suggest that $\mathrm{Nrm} 1$ is likely inactivated by the replication checkpoint resulting in dissociating from MBF at promoters and the induction of G1/S genes.

\section{Overexpression of Tos 4 halts progression of the cell cycle}

Taken together, our results reveal that TOS4 expression is under an elaborate transcriptional regulation that involves $\mathrm{SBF}$ and MBF, as well as replication checkpoint signalling. To address the functional relevance of controlling TOS4 expression, we tested the effect of TOS4 overexpression by ectopically expressing it from a GAL1-inducible system. FACS analysis of wild-type and Tos4 overexpressing cells revealed that overexpression of Tos 4 results in a significant delay in cell-cycle progression after release from an $\alpha$-factor-enforced G1 arrest (Figure 6A). This result reveals that TOS4 is a dosage-sensitive gene whose overexpression delays S-phase progression.

\section{Tos4 is essential for cell survival during replication stress in the absence of the checkpoint protein kinase Dun 1}

The strong Rad53-dependent up-regulation of Tos4 transcription in response to replication stress and the potential role of Tos4 in promoting cell-cycle arrest suggest that Tos4 is an effector of the replication checkpoint. To determine the relevance of Tos4 during replication stress, we tested the sensitivity of tos $4 \Delta$ cells to $\mathrm{HU}$ treatment. As shown in 


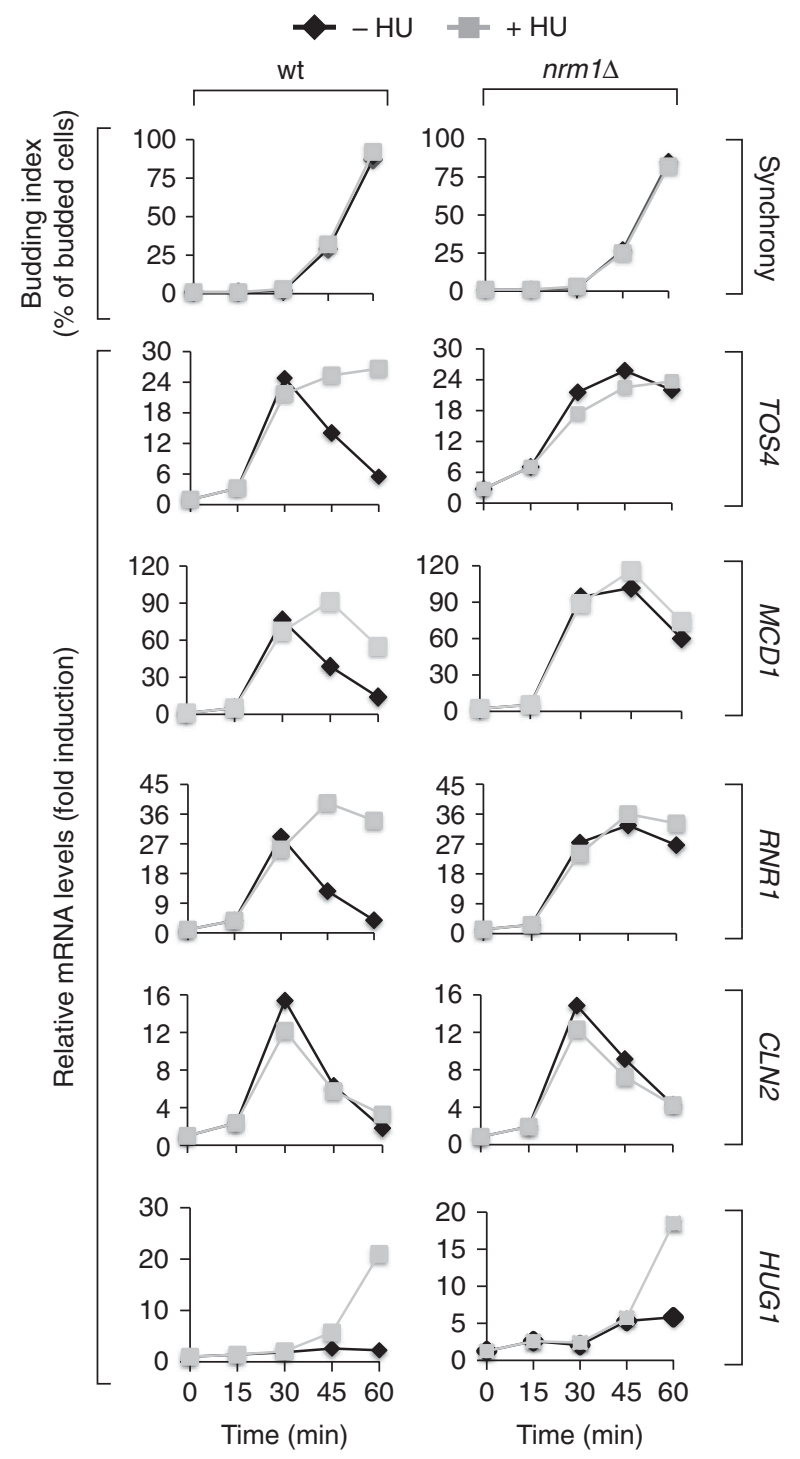

Figure $5 \mathrm{Nrm} 1$ represses expression of MBF targets during unperturbed $\mathrm{S}$ phase but not during replication stress. Relative mRNA levels of indicated genes in wild-type and $n r m 1 \Delta$ cells in the presence or absence of HU. For HU treatment, $100 \mathrm{mM}$ HU was added $20 \mathrm{~min}$ after release from $\alpha$-factor arrest. Levels of mRNA were quantified by RT-qPCR, normalized to ACT1, and presented as fold induction of levels detected in time 0 in wild-type cells. Budding index (\% budded cells) was measured as an indicator of synchrony.

Figure $6 \mathrm{~B}$, tos $4 \Delta$ cells are not sensitive to HU. However, we found that in cells lacking the downstream checkpoint kinase Dun1, Tos4 plays an essential role in cell viability in the response to replication stress, as tos $4 \Delta d u n 1 \Delta$ cells are highly sensitive to HU. This result is consistent with our finding that transcriptional regulation of Tos4 is Dun1-independent, suggesting that Tos4 represents a branch of the replication checkpoint that is parallel to the Dun1mediated response. In addition, microscopy analysis of cells expressing Tos4-GFP demonstrates that Tos4 rapidly accumulates in the nucleus upon HU treatment, consistent with its potential role in the DNA replication checkpoint (Supplementary Figure S4). Collectively, these findings support a role for Tos4 as a replication checkpoint effector.

\section{The FHA domain of Tos4 mediates an interaction with the Rpd3L and Set3 HDAC complexes to promote cellular tolerance to replication stress}

Tos4 is an FHA (ForkHead-associated) domain containing protein of unknown function. FHA domains are proteinprotein interaction motifs originally identified in the ForkHead cell-cycle transcription factors but are also found in checkpoint proteins, including Rad53 and Dun1 (Mohammad and Yaffe, 2009). In order to test weather the FHA domain of Tos4 mediates its role during replication stress response, we generated point mutations in two residues predicted to be critical for the ability of FHA domains to mediate protein-protein interactions (Figure 6C). Mutation of the FHA domain of Tos 4 in cells lacking Dun1 resulted in a HU sensitivity compared with that of tos $4 \Delta$ dun $1 \Delta$ cells (Figure 6D), suggesting that the role of Tos4 during replication stress is dependent on the ability of its FHA domain to interact with other protein(s). Protein abundance of the FHA mutant is not affected when compared with wt Tos4 (Supplementary Figure S5). Using quantitative mass spectrometry, we compared the set of proteins that specifically interact with wild-type Tos4 but not with a Tos4 mutant containing an impaired FHA domain (Supplementary Figure S6; Dataset 2 in Supplementary data). Wild-type Tos4 was found to specifically interact with several components of the histone deacetylase (HDAC) complexes Rpd3 and Set3 (Figure 6E). These data confirm previous mass spectrometry results that identified Tos 4 in $\mathrm{Rpd} 3$ and Set3 pull downs (Shevchenko et al, 2008). Our results show that this interaction is lost in the Tos4 FHA mutant. These HDACs have been shown to play an important role in the transcriptional response to various environmental stresses and more specifically in the regulation of transcription in the DDR (Sharma et al, 2007; Alejandro-Osorio et al, 2009). Tos4's interaction with these HDAC complexes suggests a possible role for Tos4 in modulating HDAC activity in response to replication stress.

Because Tos4 accumulates in response to replication stress and binds, through its FHA domain, to HDAC complexes, we hypothesized that if Tos4 is required for HDAC activity during replication stress, inactivation of the interacting HDAC components should have no effect or a negative effect on cell viability in response to replication stress in the absence of Tos4 and Dun1. In contrast, if Tos 4 interferes with HDAC function in response to replication stress, this should suppress the HU sensitivity of tos $4 \Delta$ dun $1 \Delta$ mutants. Deletion of the catalytic subunits RPD3 or HST1 was found to suppress the sensitivity of tos $4 \Delta d u n 1 \Delta$ cells to HU treatment, suggesting a potential role for Tos 4 in counteracting the activity of these HDACs components (Figure 6F). Since the FHA domain of Tos 4 is essential for its binding to the HDAC complexes, these findings suggest an important role for Tos4 in regulating HDAC function during replication stress. Collectively, these results suggest that Tos 4 is an effector of the replication checkpoint that couples Rad53 signalling to the regulation of HDACs function during replication stress.

\section{Overlapping SCB/MCB motifs characterizes a distinct group of $\mathrm{G1} / \mathrm{S}$ genes}

Our results reveal that an SBF-to-MBF switch regulates the expression of TOS4, an effector of the replication checkpoint. Analysis of the promoter of other G1/S genes found to be strongly up-regulated by $\mathrm{HU}$ in our proteomic screen revealed 
A

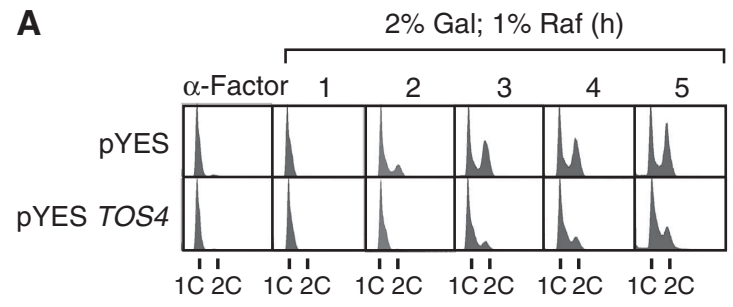

C

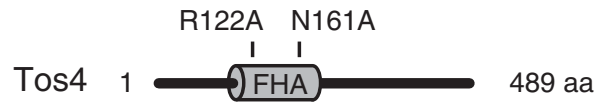

B

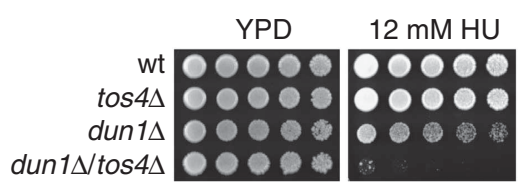

E

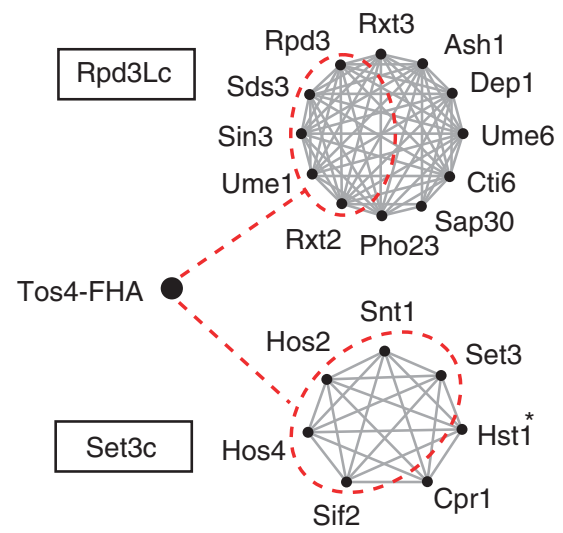

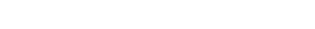

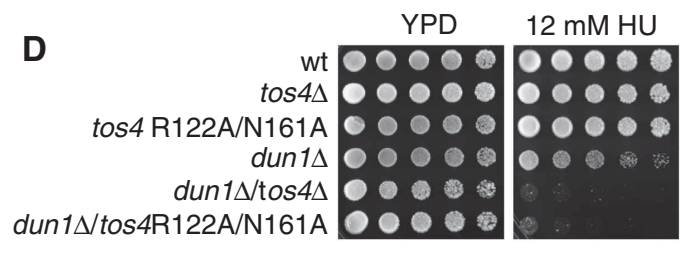

$\mathbf{F}$

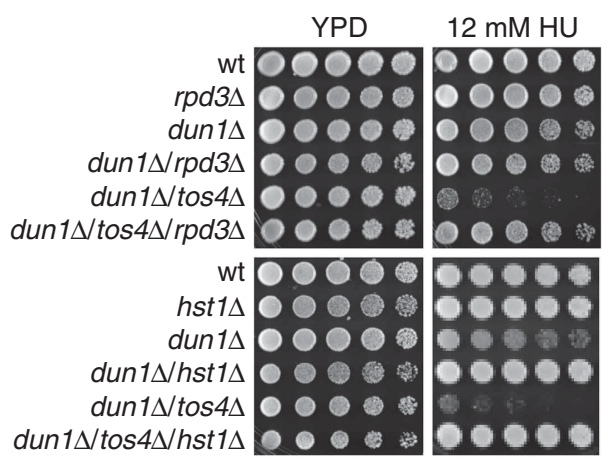

Figure 6 Tos4 plays an important role in the replication stress response via interaction with HDACs. (A) FACS analysis of cells overexpressing TOS4 during S phase. Cells were synchronized in G1 by $\alpha$-factor arrest and released from arrest in $2 \%$ galactose $1 \%$ raffinose for the indicated interval. (B) HU sensitivity of indicated strains. Four-fold serial dilutions were spotted on plates and grown for $2-3$ days at $30^{\circ} \mathrm{C}$. (C) Schematic representation of Tos4 protein displaying its FHA domain and the position of residues that were mutated to disrupt FHA domain function. (D) HU sensitivity of indicated strains. Four-fold serial dilutions were spotted on plates and grown for $2-3$ days at $30^{\circ} \mathrm{C}$. (E) Network of Tos4 FHA-interacting proteins during replication stress. According to available information in the Biogrid Database (http://thebiogrid.org/), the identified interacting proteins were grouped into two distinct complexes represented by the Rpd3 and Set3 HDACs. Grey lines represent previously known interactions between the different subunits that comprise both HDACs. Dotted line indicates the interactions identified in this work. ( $\left.{ }^{*}\right)$ Peptides for Hst1 were not detected in the MS experiment, but the interaction was validated by co-IP (see Supplementary Figure S6). (F) HU sensitivity of indicated strains. Four-fold serial dilutions were spotted on plates and grown for $2-3$ days at $30^{\circ} \mathrm{C}$.

that the promoter of $M C D 1$ also contains overlapping $\mathrm{SCB}$ / MCB motifs (Figure 7A). Mcd1 is an essential subunit of cohesin that prevents chromosome segregation and whose overexpression arrests cells in G2/M (Sopko et al, 2006). We further confirmed that, like TOS4, MCD1 expression is also regulated by an SBF-to-MBF switch (Figure 7B-D). Next, we sought to determine how common the SBF-to-MBF switch regulation is for G1/S targets. To identify putative SBF/MBF switching promoters, we analysed the promoter sequences of the $200+$ SBF and MBF target genes identified in the Iyer ChIP-chip study and the Simon et al (2001) and Harbison et al (2004) data sets (Iyer et al, 2001) for the presence of overlapping SCB/MCB motifs. We classified a target promoter to contain an overlapping SCB/MCB sites as when an SCB and $\mathrm{MCB}$ sequence overlap, maintaining both consensus binding sequences (SCB; CNCGAAA and MCB; ACGCG). The analysis of Iyer ChIP-chip data set identified $27 \mathrm{SBF} / \mathrm{MBF}$ target promoters with one or more overlapping SCB/MCB motifs (Supplementary Table SI). Analysis of the two data sets from the Young laboratory (Simon et al, 2001; Harbison et al, 2004) identified 32 and 22 genes containing overlapping SCB/MCB motifs, respectively but did not identify the founding switch gene member TOS4. Since our study shows that TOS4 is a genuine SBF/MBF target and cell cycle regulated gene, we used the list from the Iyer data set for the initial study of this new group of G1/S genes. A more comprehensive list of potential switch genes from all three data sets, containing 44 genes is included (Supplementary Table SI). Interestingly, the majority of these promoters were designated as SBF targets, with a few for both and none as MBF-only targets. We selected six of the targets containing overlapping SCB/MCB motif to further test by ChIP analysis if they are indeed regulated by SBF/MBF switch. As shown in Figure 7E, in all cases we were able to confirm the switch. Next, we analysed the combined genome-wide overexpression data sets from studies by Sopko et al (2006) and Yoshikawa et al (2011) for total genes tested, G1/S transcripts (based on Iyer et al, data set) and switch genes. In line with our hypothesis, the switch genes are significantly enriched for genes that cause a cell cycle delay or growth defect when overexpressed (Figure 7F). Finally, we also analysed the HU transcriptional regulation of 27 switch genes defined in this work. Our result shows that $2 / 3$ of the tested switch genes are up-regulated upon HU treatment (Supplementary Table SI; Supplementary Figure S7). Taken 
A

MCD1 promoter elements

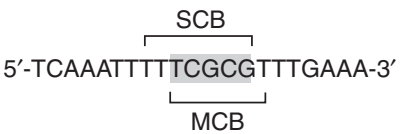

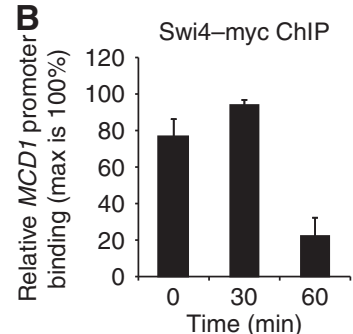

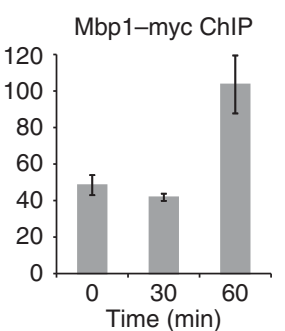

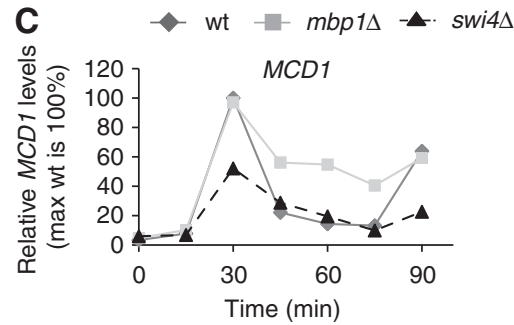

E

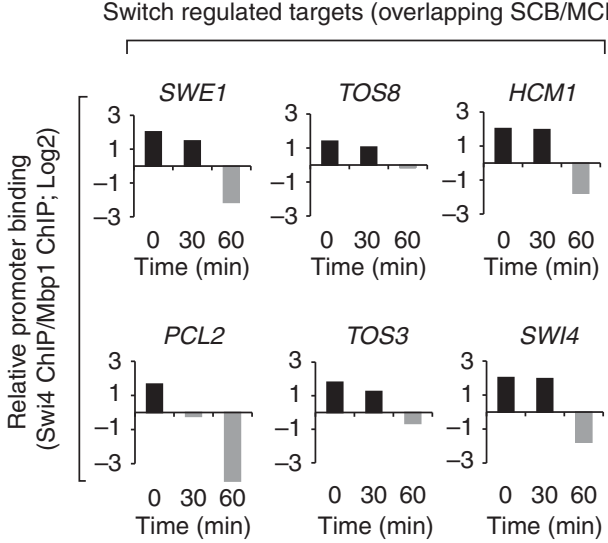

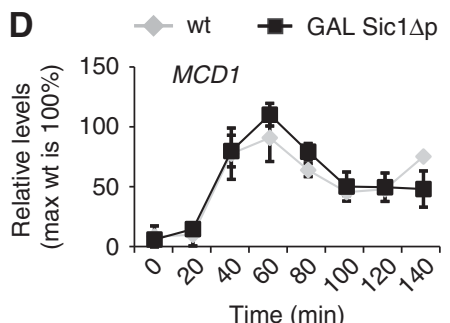

$\mathbf{F}$

Genome-wide (5854)

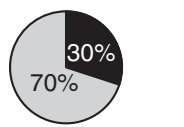

G1/S (172)

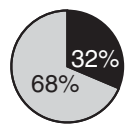

Switch (27)

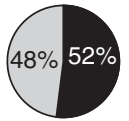

Dosage-sensitive genes shown by Sopko et al and Yoshikawa et al

Figure 7 SBF-to-MBF switch at the promoter of other G1/S genes. (A) Schematic representation of overlapping SCB and MCB motifs identified in the $M C D 1$ promoter element ( -336 to -329 from the ATG start codon). Grey shade represents SCB/MCB overlapping region. (B) ChIP analysis for Swi4-myc or Mbp1-myc at the MCD1 promoter during the G1 and S phase of the cell cycle. Experiment was performed as described in Figure 3A. (C, D) Relative mRNA levels of MCD1 during the cell cycle in (C) wild-type, swi4 $\triangle$ and mbp1 $\Delta$ cells and (D) wild-type and cells expressing sic $1 \Delta \mathrm{p}$ from the inducible GAL1 promoter. Experiments were performed as described in Figures 3B and 4D, respectively. (E) ChIP analysis for Swi4-myc or Mbp1-myc at the promoter of the indicated genes. Analysis was performed in G1 synchronized cells released from $\alpha$-factor arrest and samples were taken at the indicated intervals. G1: 0 min, G1/S: $30 \mathrm{~min}$ and S phase: 60 min after release. Enrichment levels were accessed by qPCR and are presented as the ratio between Swi4-myc/Mbp1-myc percentages after normalization of each ChIP for its corresponding maximum value. (F) Percentage (\%) of dosage-sensitive genes as compared with the number of genes tested in the combined genome-wide overexpression studies of Sopko et al (2006) and Yoshikawa et al (2011) for the indicated gene categories. The number of genes tested for overexpression in each of the categories is indicated in parenthesis.

together, these data suggest that the SBF to MBF switch mechanism defines a new group of G1/S genes enriched for dosagesensitive genes and/or replication stress-induced genes.

\section{Discussion}

Here, we report that increase in the expression of $\mathrm{G} 1 / \mathrm{S}$ cell-cycle genes is a prominent part of the transcriptional response to replication stress, and we uncover the mechanisms governing such transcriptional up-regulation. We found that replication stress induces transcription of $\mathrm{MBF}$, but not SBF targets in a Rad53-dependent manner likely via inactivation of the MBF co-repressor Nrm1. A more comprehensive study, which establishes the molecular mechanism of this regulation, has been carried out in the Wittenberg laboratory (Travesa et al, 2012). We show that this pathway functions in parallel to the Rad53-Dun1-dependent inhibition of the Crt1 repressor, a pathway that mediates induction of DDR genes (Figure 8A). Furthermore, we uncover a novel SBF-to-MBF switch mechanism that allows G1/S genes targeted by SBF during G1 to be regulated by MBF once cells progress into $\mathrm{S}$ phase, which allows regulation by the replication checkpoint (Figure 8B). To our knowledge, this is the first evidence of SBF/MBF switching during the cell cycle at a G1/S promoter. The importance of this mechanism of regulation is illustrated by our detailed analysis of the 'switch gene' TOS4, which shows the importance of its induction in response to replication stress and its toxicity when overexpressed. We propose that the SBF-to-MBF switch is a mechanism of transcriptional control that allows checkpoint 
A

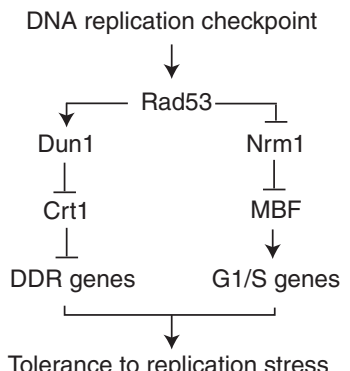

B

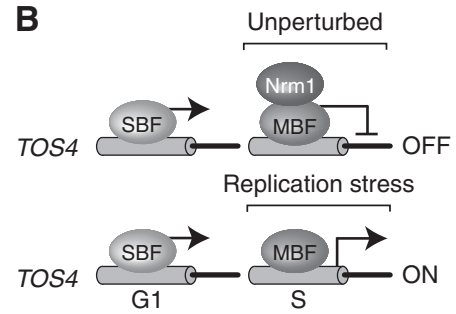

Figure 8 (A) Parallel pathways for the replication checkpoint-dependent transcriptional regulation in budding yeast. Upon replication stress, Rad53 induces the expression of DDR genes via Dun1/ Crt1 pathway and de-represses transcription of MBF-dependent targets required for replication stress response. (B) Regulation of TOS4 as a prototypical example of SBF-to-MBF subunit switch. TOS4 transcription is regulated via subunit switching and replication checkpoint signalling. During G1, SBF binds to TOS4 promoter and activates transcription. Upon entry into S phase, SBF dissociates from the TOS4 promoter in a Clb/CDK1-dependent manner leaving TOS4 promoter available for binding of MBF and its corepressor Nrm1. In response to replication stress, Nrm1 dissociates from $\mathrm{MBF}$ in response to replication checkpoint activation leading to transcriptional de-repression of TOS4. (C) Proposed importance of SBF-to-MBF subunit switching. The SBF/MBF switch on G1/S promoters provides a fail-safe mechanism that prevents loss of periodicity and hyperaccumulation of dosage-sensitive genes during G1 in case of MBF loss of function. $\left({ }^{*}\right)$ Indicate loss of periodicity in case of MBF 'malfunction'

activation but prevents loss of periodicity and potential hyperaccumulation of dosage-sensitive proteins in case of cell-cycle transcription factor failure (Figure 8C). Overall, our work demonstrates how the coordinated action of SBF and MBF during the cell cycle allows for the crosstalk between transcription of G1/S genes and the replication checkpoint upon replication stress.

\section{MBF as a link between replication checkpoint signalling and transcriptional regulation of $\mathrm{G1} / \mathrm{S}$ genes}

We found that the accumulation of MBF targets upon replication stress involves Rad53-dependent regulation of the MBF co-repressor Nrm1. Similar observations have been made, using a genome-wide transcriptional approach, by the Wittenberg laboratory (Travesa et al, 2012). Our findings provide evidence for this regulation to be conserved between the distantly related budding and fission yeast, Schizosaccharomyces pombe. In fission yeast, the G1/S transcription depends on a single heterodimeric transcription factor complex, which is, based on its homology to budding yeast, also called MBF (Lowndes et al, 1992; Caligiuri and Beach, 1993; Whitehall et al, 1999). However, in addition to $\mathrm{Nrm} 1$, the transcriptional repressor Yox1 is also required to confine transcription to the G1 phase (Aligianni et al, 2009). It has been shown that in response to replication stress, MBFdependent transcription is activated via checkpoint protein kinase Cds1 phosphorylation-dependent inactivation of both Nrm1 and Yox1 (de Bruin et al, 2008; Caetano et al, 2011; Gomez-Escoda et al, 2011; Purtill et al, 2011). Overall, our study shows that the mechanism that governs the remodelling of the cell-cycle transcriptional programme by the DNA replication checkpoint is conserved between these distantly related yeasts.

\section{SBF-to-MBF switch genes; a new group of G1/S genes} Detailed analysis of the transcriptional regulation of the G1/S gene TOS4 allowed us to uncover a prototypical SBF-to-MBF switch at a promoter gene in yeast. We speculate that overlapping SCB and $\mathrm{MCB}$ elements at gene promoters is likely the basis for the switch, only allowing MBF or SBF to be bound at any given time. The presumed higher binding affinity of SBF for the SCB element would allow SBF to compete for the binding during G1. On the other hand, our data show that switch genes are inactivated in a timely manner even in the absence of $\mathrm{Clb} / \mathrm{CDK}$ activity, suggesting that MBF actively replaces SBF at switch promoters during $\mathrm{S}$ phase. In addition, the data show that the non-overlapping target gene CLN1 remains active when Clb/CDK activity is inhibited. This together with $C L N 1$ being repressed in $m b p 1 \Delta$ cells during $S$ phase and in response to $\mathrm{HU}$ treatment (Supplementary Figure S8) and being bound my MBF throughout the cell cycle shows a clear difference in regulation between an dual-regulated SBF and MBF target (i.e., CLN1) and 'switch' genes (i.e., TOS4 and MCD1), establishing the latter as a new group of G1/S genes.

\section{Rationale for an SBF-to-MBF switch for the regulation of G1/S genes}

Analysis of $200+$ G1/S promoters, identified by ChIP-chip (Iyer et al, 2001), resulted in the identification of a total of 27 genes with an overlapping SCB/MCB motif, suggesting that SBF/MBF switching might be a common mode of regulation. SBF- and MBF-regulated genes appear to be segregated based upon their role in the cell. Whereas the majority of essential G1/S genes are regulated by MBF (Iyer et al, 2001) non-essential genes involved in the timing or efficiency of cell-cycle events are more likely to be regulated by SBF. This way, MBF malfunction results in viable cells, constitutively expressing essential genes, while SBF malfunction will not result in uncontrolled cell proliferation with low expression of genes involved in cell cycle timing. So when is regulation by an SBF-to-MBF switch advantageous? We speculate that $\mathrm{G} 1 / \mathrm{S}$ genes that need to be induced in response to replication stress, but whose constitutive expression is detrimental to cell-cycle progression, would benefit from a regulation by a SBF/MBF switch. For example, the SBF/MBF switch allows TOS4 to behave as a replication stress-inducible gene during $\mathrm{S}$ phase but avoids constitutive high expression and hyperaccumulation of Tos4 in case MBF function is compromised (Figure 8C). Consistent with this notion, we show that Tos 4 has an important role in the checkpoint response and that overexpression of Tos4 delays cell-cycle progression. Analysis of the cluster of genes with overlapping $\mathrm{SCB} / \mathrm{MCB}$ motifs revealed that overexpression of most of those genes has been reported to result in cell cycle delay and/or growth defect. Moreover, analysis of transcription induction upon HU treatment showed that this group of $\mathrm{G} 1 / \mathrm{S}$ switch genes is enriched by genes that are 
up-regulated during replication stress conditions. Taken together, the results reveal that SBF-to-MBF switch preferentially regulates dosage-sensitive genes, and we propose that most of the genes controlled by the switch play important roles during replication stress response, as shown here for Tos4, and previously for Mcd1 and Swe1 (Strom et al, 2004; Enserink et al, 2006; Liu and Wang, 2006; Covo et al, 2010). Further functional analysis of the other genes regulated by the $\mathrm{SBF} / \mathrm{MBF}$ switch, most of which are currently annotated as putative genes, may reveal other dosage-sensitive genes and/or yet undescribed checkpoint effectors.

\section{Rad53-MBF-Tos4 pathway; a novel branch of the replication checkpoint}

Tos4 was originally identified as an SBF target and a putative transcription factor suggested to repress pheromone response genes during the cell cycle and in the presence of $\alpha$-factor (Horak et al, 2002). Several lines of evidence presented here support that Tos4 is an effector of the replication checkpoint. First, the expression of Tos4 is strongly up-regulated during replication stress in a Rad53-dependent manner. Second, following replication stress, Tos4 rapidly accumulates in the nucleus. Third, overexpression of Tos 4 causes a cell cycle delay. Fourth, Tos4 contains an FHA domain, commonly found in checkpoint proteins, that is required for its function. Furthermore, we found that Tos4 plays a parallel role to Dun1 in the replication checkpoint. This is supported by the findings that transcription of TOS4 is regulated in a Dun1independent manner and that tos $4 \Delta d u n 1 \Delta$ cells exhibit a much stronger HU sensitivity as compared with the single deletes. Together, our findings establish that the Rad53-MBFTos4 pathway represents a novel branch of the replication checkpoint (Figure 8A). While the exact role of Tos4 remains unclear, the ability of Tos4 to interact with the Rpd3 and Set3 HDAC complexes and the importance of this interaction for the survival of cells in response to replication stress suggests that Tos4 functions to couple replication checkpoint signalling to the regulation of HDAC function.

\section{Comparisons with mammalian G1/S transcription}

Our work suggests that G1/S transcription factor switching assures that malfunction of any one transcription factor does not result in complete loss of periodicity (Figure 8C). In mammalian cells, switching of the E2F transcription factors at G1/S promoters during the cell cycle seems to be the standard (Dimova and Dyson, 2005; Moon and Dyson, 2008), suggesting that loss of periodicity is detrimental. In line with this, the high frequency of genetic alterations in genes involved in E2F-dependent G1/S transcriptional regulation found in human tumour cells indicates an important role for proper regulation of the E2F system in the prevention of tumour development (Stevens and La Thangue, 2003).

In addition, we uncovered in yeast a simple but elegant mechanism by which checkpoint activation can override the G1/S transcriptional programme by directly regulating a transcriptional repressor. In human cells, the main regulator of DNA damage-inducible genes in G1 is the transcription factor p53, a target of both ATM and Chk2 (Carr, 2000). However, the transcriptional response induced by the DNA replication checkpoint in S phase is regulated largely through a p53-independent mechanism that has yet to be fully elucidated. Interestingly, recent data suggest that, in response to DNA replication stress, like in yeast, G1/S transcription, regulated by the E2F family of transcription factors, is maintained at high levels in a Chk1-dependent manner (Cosetta Bertoli and RAM de Bruin, unpublished data).

If, as preliminary data suggest, remodelling of the G1/S cell-cycle transcriptional programme by the DNA replication checkpoint proves to be conserved from yeast to man, this would represent the largest group of co-regulated genes among DNA replication stress-induced targets and, consequently, is likely to comprise an important mechanism for the avoidance of genomic instability in human cells. It will be important to establish the precise mechanism of this control in human cells and the importance of this regulation for genome integrity in eukaryotes.

\section{Materials and methods}

\section{Yeast strains and plasmids}

Strains used in this work were generated by standards genetics methods and derived from S288C (MATa, ura3-52, trp1-63, his3200), MBS164-YPH499, congenic to S288C (MATa, ura3-52, leu2 $\Delta 1$, trp1-63, his3-200, lys2 $\Delta B g l$, hom3-10, ade2 $\Delta 1$, ade8, arg4 $\Delta$, sml1::TRP bar1::HIS3) or 15Daub (MATa, ade1, leu2-3, 112 his2, trp1-1, ura3 $\Delta$ ns, bar1 $\Delta$ ) unless otherwise stated. All plasmids and yeast strains used in this study are described on Supplementary data (Supplementary Tables SII and SIII, respectively). Strains and plasmids are available upon request.

\section{Cell synchronization}

Mating pheromone arrest synchrony experiments were also carried out as described (Stuart and Wittenberg, 1995). In the experiments involving strains carrying $G A L-\operatorname{sic} 1 \Delta \mathrm{p}$, an overnight culture grown in $2 \%$ raffinose $0.2 \%$ glucose was used to inoculate a $2 \%$ raffinose culture. This culture was grown for $2 \mathrm{~h}$ before mating pheromone was added. Two hours after mating pheromone addition, $2 \%$ galactose was added and $2 \mathrm{~h}$ later cells were released from the G1 arrest in $2 \%$ galactose medium and samples were taken.

\section{SILAC labelling of yeast and mass spectrometry analysis}

For mass spectrometry experiments, cells were grown in (-) Arg (-) Lys dropout media ('light' version complemented with normal arginine and lysine; 'heavy' version complemented with lysine ${ }^{13} \mathrm{C} 6,{ }^{15} \mathrm{~N} 2$ and arginine ${ }^{13} \mathrm{C} 6,{ }^{15} \mathrm{~N} 4$ ) (see Supplementary data for details). Proteins were digested with trypsin, peptides were desalted, dried, reconstituted in $80 \%$ acetonitrile and $1 \%$ formic acid and fractionated by hydrophilic interaction chromatography (HILIC) as previously described in Albuquerque et al (2008). Fractions were dried, reconstituted in $0.1 \%$ trifluoroacetic acid and analysed by LC-MS/MS using $125 \mu \mathrm{M}$ ID capillary C18 column and an Orbitrap XL mass spectrometer coupled with and Eksigent nanoflow system. Database search and analysis was performed as previously described in Ohouo et al (2010).

\section{Reverse transcriptase (RT) and quantitative (q)PCR}

Total RNA was isolated using the Rneasy kit (Qiagen). The QuantiTech $^{\mathrm{TM}}$ SYBR® Green PCR kit (Qiagen) was used for quantitative PCR on ChIP samples and the QuantiTech SYBR Green RT-PCR kit (Qiagen) was used for RT-PCR experiments. Reactions were run on the Chromo-4 qPCR I system (MJ Research) using standard PCR and RT/PCR conditions. Data were analysed using MJ Opticon Monitor Analysis Software 3.0.

\section{ChIP analysis}

Chromatin immunoprecipitation was performed as described (Flick et al, 2003). 


\section{Supplementary data}

Supplementary data are available at The EMBO Journal Online (http://www.embojournal.org).

\section{Acknowledgements}

We thank Steffi Klier and Inesa Rozenman from the de Bruin laboratory and Beatriz Almeida from the Smolka laboratory for technical assistance. We thank J Skotheim and J Bähler for comments on the manuscript and members of the MRC LMCB for helpful discussion. Thanks to the Wittenberg laboratory for sharing results before publication. FMBdO was supported by a Cornell

\section{References}

Albuquerque CP, Smolka MB, Payne SH, Bafna V, Eng J, Zhou H (2008) A multidimensional chromatography technology for in-depth phosphoproteome analysis. Mol Cell Proteomics 7: $1389-1396$

Alejandro-Osorio AL, Huebert DJ, Porcaro DT, Sonntag ME, Nillasithanukroh S, Will JL, Gasch AP (2009) The histone deacetylase Rpd3p is required for transient changes in genomic expression in response to stress. Genome Biol 10: R57

Aligianni S, Lackner DH, Klier S, Rustici G, Wilhelm BT, Marguerat S, Codlin S, Brazma A, de Bruin RA, Bahler J (2009) The fission yeast homeodomain protein Yox $1 p$ binds to MBF and confines MBF-dependent cell-cycle transcription to G1-S via negative feedback. PLoS Genet 5: e1000626

Allen JB, Zhou Z, Siede W, Friedberg EC, Elledge SJ (1994) The SAD1/RAD53 protein kinase controls multiple checkpoints and DNA damage-induced transcription in yeast. Genes Dev 8: 2401-2415

Amon A, Tyers M, Futcher B, Nasmyth K (1993) Mechanisms that help the yeast cell cycle clock tick: G2 cyclins transcriptionally activate G2 cyclins and repress G1 cyclins. Cell 74: 993-1007

Bean JM, Siggia ED, Cross FR (2005) High functional overlap between MluI cell-cycle box binding factor and Swi4/6 cell-cycle box binding factor in the G1/S transcriptional program in Saccharomyces cerevisiae. Genetics 171: 49-61

Breeden LL (2003) Periodic transcription: a cycle within a cycle. Curr Biol 13: R31-R38

Caetano C, Klier S, de Bruin RA (2011) Phosphorylation of the MBF repressor Yox1p by the DNA replication checkpoint keeps the G1/S cell-cycle transcriptional program active. PLoS One 6: e17211

Caligiuri M, Beach D (1993) Sct1 functions in partnership with Cdc10 in a transcription complex that activates cell cycle START and inhibits differentiation. Cell 72: 607-619

Carr AM (2000) Cell cycle piecing together the p53 puzzle. Science 287: $1765-1766$

Chen HZ, Tsai SY, Leone G (2009) Emerging roles of E2Fs in cancer: an exit from cell cycle control. Nat Rev Cancer 9: 785-797

Cobrinik D (2005) Pocket proteins and cell cycle control. Oncogene 24: $2796-2809$

Costanzo M, Nishikawa JL, Tang X, Millman JS, Schub O, Breitkreuz K, Dewar D, Rupes I, Andrews B, Tyers M (2004) CDK activity antagonizes Whi5, an inhibitor of G1/S transcription in yeast. Cell 117: 899-913

Covo S, Westmoreland JW, Gordenin DA, Resnick MA (2010) Cohesin is limiting for the suppression of DNA damage-induced recombination between homologous chromosomes. PLoS Genet 6: e1001006

de Bruin RA, Kalashnikova TI, Chahwan C, McDonald WH, Wohlschlegel J, Yates 3rd J, Russell P, Wittenberg C (2006) Constraining G1-specific transcription to late G1 phase: the MBF-associated corepressor Nrm1 acts via negative feedback. Mol Cell 23: 483-496

de Bruin RA, Kalashnikova TI, Chahwan C, Wohlschlegel J, Yates 3rd JR, Russell P, Wittenberg C (2008) The DNA replication checkpoint regulates G1/S dependent transcription via phosphorylation of the MBF-bound repressor, Nrm1. Proc Nat Acad USA 105: $11230-11235$

de Bruin RA, McDonald WH, Kalashnikova TI, Yates 3rd J, Wittenberg C (2004) Cln3 activates G1-specific transcription
Fleming Research Fellowship. RAMdB, MRH and PB were funded by the MRC. This work was supported by Research Scholar Grant \#RSG-11-146-01-DMC from the American Cancer Society to MBS.

Author contributions: FMBdO, MRH, RAMdB and MBS designed the research; FMBdO, MRH, RAMdB, PB and MBS performed the research; FMBdO, MRH, RAMdB and MBS analysed the data; and FMBdO, RAMdB and MBS wrote the paper.

\section{Conflict of interest}

The authors declare that they have no conflict of interest. via phosphorylation of the SBF bound repressor Whi5. Cell 117: 887-898

Dimova DK, Dyson NJ (2005) The E2F transcriptional network: old acquaintances with new faces. Oncogene 24: 2810-2826

Enserink JM, Smolka MB, Zhou H, Kolodner RD (2006) Checkpoint proteins control morphogenetic events during DNA replication stress in Saccharomyces cerevisiae. J Cell Biol 175: 729-741

Eser U, Falleur-Fettig M, Johnson A, Skotheim JM (2011) Commitment to a cellular transition precedes genome-wide transcriptional change. Mol Cell 43: 515-527

Flick KM, Spielewoy N, Kalashnikova TI, Guaderrama M, Zhu Q, Chang HC, Wittenberg C (2003) Grr1-dependent inactivation of Mth1 mediates glucose-induced dissociation of Rgt1 from HXT gene promoters. Mol Biol Cell 14: 3230-3241

Frolov MV, Dyson NJ (2004) Molecular mechanisms of E2Fdependent activation and pRB-mediated repression. J Cell Sci 117(Pt 11): 2173-2181

Fu Y, Pastushok L, Xiao W (2008) DNA damage-induced gene expression in Saccharomyces cerevisiae. FEMS Microbiol Rev 32: 908-926

Gasch AP, Huang M, Metzner S, Botstein D, Elledge SJ, Brown PO (2001) Genomic expression responses to DNA-damaging agents and the regulatory role of the yeast ATR homolog Mec1p. Mol Biol Cell 12: 2987-3003

Gomez-Escoda B, Ivanova T, Calvo IA, Alves-Rodrigues I, Hidalgo E, Ayte J (2011) Yox1 links MBF-dependent transcription to completion of DNA synthesis. EMBO Rep 12: 84-89

Harbison CT, Gordon DB, Lee TI, Rinaldi NJ, Macisaac KD, Danford TW, Hannett NM, Tagne JB, Reynolds DB, Yoo J, Jennings EG, Zeitlinger J, Pokholok DK, Kellis M, Rolfe PA, Takusagawa KT, Lander ES, Gifford DK, Fraenkel E, Young RA (2004) Transcriptional regulatory code of a eukaryotic genome. Nature 431: 99-104

Horak CE, Luscombe NM, Qian J, Bertone P, Piccirrillo S, Gerstein M, Snyder M (2002) Complex transcriptional circuitry at the G1/S transition in Saccharomyces cerevisiae. Genes Dev 16: 3017-3033

Huang M, Zhou Z, Elledge SJ (1998) The DNA replication and damage checkpoint pathways induce transcription by inhibition of the Crt1 repressor. Cell 94: 595-605

Iyer VR, Horak CE, Scafe CS, Botstein D, Snyder M, Brown PO (2001) Genomic binding sites of the yeast cell-cycle transcription factors SBF and MBF. Nature 409: 533-538

Liu H, Wang Y (2006) The function and regulation of budding yeast Swe1 in response to interrupted DNA synthesis. Mol Biol Cell 17: 2746-2756

Lopes M, Cotta-Ramusino C, Pellicioli A, Liberi G, Plevani P, Muzi-Falconi M, Newlon CS, Foiani M (2001) The DNA replication checkpoint response stabilizes stalled replication forks. Nature 412: 557-561

Lowndes NF, McInerny CJ, Johnson AL, Fantes PA, Johnston LH (1992) Control of DNA synthesis genes in fission yeast by the cellcycle gene cdc10 + . Nature 355: 449-453

Mohammad DH, Yaffe MB (2009) 14-3-3 proteins, FHA domains and BRCT domains in the DNA damage response. DNA Repair 8: 1009-1017

Moon NS, Dyson N (2008) E2F7 and E2F8 keep the E2F family in balance. Dev Cell 14: 1-3

Ohouo PY, Bastos de Oliveira FM, Almeida BS, Smolka MB (2010) DNA damage signaling recruits the Rtt107-Slx4 scaffolds via Dpb11 to mediate replication stress response. Mol Cell 39: 300-306 
Purtill FS, Whitehall SK, Williams ES, McInerny CJ, Sharrocks AD, Morgan BA (2011) A homeodomain transcription factor regulates the DNA replication checkpoint in yeast. Cell Cycle 10: 664-670

Segurado M, Tercero JA (2009) The S-phase checkpoint: targeting the replication fork. Biol Cell 101: 617-627

Sharma VM, Tomar RS, Dempsey AE, Reese JC (2007) Histone deacetylases RPD3 and HOS2 regulate the transcriptional activation of DNA damage-inducible genes. Mol Cell Biol 27: 3199-3210

Shevchenko A, Roguev A, Schaft D, Buchanan L, Habermann B, Sakalar C, Thomas H, Krogan NJ, Stewart AF (2008) Chromatin central: towards the comparative proteome by accurate mapping of the yeast proteomic environment. Genome Biol 9: R167

Simon I, Barnett J, Hannett N, Harbison CT, Rinaldi NJ, Volkert TL, Wyrick JJ, Zeitlinger J, Gifford DK, Jaakkola TS, Young RA (2001) Serial regulation of transcriptional regulators in the yeast cell cycle. Cell 106: 697-708

Sopko R, Huang D, Preston N, Chua G, Papp B, Kafadar K, Snyder M, Oliver SG, Cyert M, Hughes TR, Boone C, Andrews B (2006) Mapping pathways and phenotypes by systematic gene overexpression. Mol Cell 21: 319-330

Stevens C, La Thangue NB (2003) E2F and cell cycle control: a double-edged sword. Arch Biochem Biophys 412: 157-169

Strom L, Lindroos HB, Shirahige K, Sjogren C (2004) Postreplicative recruitment of cohesin to double-strand breaks is required for DNA repair. Mol Cell 16: 1003-1015
Stuart D, Wittenberg C (1995) CLN3, not positive feedback, determines the timing of CLN2 transcription in cycling cells. Genes Dev 9: 2780-2794

Tercero JA, Diffley JF (2001) Regulation of DNA replication fork progression through damaged DNA by the Mec1/Rad53 checkpoint. Nature 412: 553-557

Travesa A, Kuo D, de Bruin RAM, Kalashnikova TI, Guaderrama M, Thai K, Aslanian A, Smolka MB, Yates III JR, Ideker T, Wittenberg C (2012) DNA replication stress differentially regulates G1/S genes via Rad53-dependent inactivation of Nrm1. EMBO J 31: 1811-1822

Whitehall S, Stacey P, Dawson K, Jones N (1999) Cell cycleregulated transcription in fission yeast: Cdc10-Res protein interactions during the cell cycle and domains required for regulated transcription. Mol Biol Cell 10: 3705-3715

Wittenberg C, Reed SI (2005) Cell cycle-dependent transcription in yeast: promoters, transcription factors, and transcriptomes. Oncogene 24: 2746-2755

Yoshikawa K, Tanaka T, Ida Y, Furusawa C, Hirasawa T, Shimizu H (2011) Comprehensive phenotypic analysis of single-gene deletion and overexpression strains of Saccharomyces cerevisiae. Yeast 28: 349-361

Zhao X, Chabes A, Domkin V, Thelander L, Rothstein R (2001) The ribonucleotide reductase inhibitor Sml1 is a new target of the Mec1/Rad53 kinase cascade during growth and in response to DNA damage. EMBO J 20: 3544-3553

Zhou Z, Elledge SJ (1993) DUN1 encodes a protein kinase that controls the DNA damage response in yeast. Cell 75: 1119-1127 